\title{
Flexible Lithium Metal Anode Featuring Ultrahigh Current Density Stability with Uniform Deposition and Dissolution
}

\author{
Ahmed M. Hafez, Jinzhi Sheng, ${ }^{1}$ Daxian Cao, ${ }^{1}$ Yong Chen ${ }^{1}$ and Hongli Zhu' ${ }^{1 *}$
}

Lithium (Li) metal anode attracted tremendous attention nowadays as a promising electrode for the next generation of rechargeable batteries. However, the uncontrollable volume change and severe dendrite formation raise safety concerns that hindered the implementation of Li metal anodes in rechargeable batteries for decades. Herein, we introduce a novel stable and flexible Li metal felt anode using thermal Li infiltration method. The structure revealed unprecedented cycling stability for 500 cycles under ultra-high current density of $40 \mathrm{~mA} \mathrm{~cm}^{-2}$. Also, it sustained its mechanical flexibility even after Li infiltration. Finite element multiphysics models were demonstrated, which illustrate in detail the fundamentals behind the outstanding stability of the Li felt. Moreover, the electrochemical full cell test comprising Li felt as anode and $\mathrm{LiFePO}_{4}$ as cathode, exhibited 7-fold higher discharging capacity of $70 \mathrm{~mA} \mathrm{~h} \mathrm{~g}$ at $10 \mathrm{C}$, corresponding to only $10 \mathrm{~mA} \mathrm{~h} \mathrm{~g}^{-1}$ for Li foil. On the other hand, the method reported in this study allows the entire process to be easily scaled up for industrial scale with roll-to-roll manufacturing. We believe that this novel structure will pave a new avenue towards promoting the performance of Li metal rechargeable batteries and hence making it finally practical for commercial applications.

Keywords: Lithium metal, Ultrahigh current density; Dendrite suppression; Ionic diffusion; Charge distribution; Finite element multiphysics

Received 24 April 2019, Accepted 28 June 2019

DOI: $10.30919 / \mathrm{esee} 8 \mathrm{c} 311$

\section{Introduction}

The rapid progress nowadays in high power density and flexible wearable electrical devices is innovating new products that are changing our daily life. These include electric vehicles, portable electronics, smart clothes, bendable smart phones, implanted medical devices, and other products that attracted tremendous attention in recent years. ${ }^{1.4}$ In order to meet the power demand for such devices, high energy and light weight storage devices are strongly required. ${ }^{5-7}$ Currently, lithium ion batteries (LIBs) are the most widely used energy storage devices in this regard, attributed to their scalability and relatively long life-cycle. ${ }^{8,9}$ However, LIBs have limited energy density that cannot support the currently high power density demand. Moreover, LIBs are usually fabricated based on current collectors with limited flexibility, which hinder their usage in flexible storage devices. This lead to the incorporation of electrochemically inactive materials in order to achieve flexibility, which further decrease the active material mass loading and affect the overall cell performance..$^{10}$ As a result, the reliability of LIBs in highly power as well as in flexible electrical devices became challenging. ${ }^{71}$

Recently, Li metal anode batteries are considered a promising alternative to LIBs. This is attributed to Li metal high specific capacity $\left(3860 \mathrm{~mA} \mathrm{~h} \mathrm{~g}^{-1}\right)$, light weight $\left(0.53 \mathrm{~g} \mathrm{~cm}^{-3}\right)$, and low electrochemical potential $\left(-3.04 \mathrm{~V}\right.$ versus the standard hydrogen electrode). ${ }^{12,13}$ However,

\footnotetext{
Department of Mechanical and Industrial Engineering, Northeastern

University, Boston, MA 02115, USA

*E-mail: h.zhu@neu.edu
}

pristine Li metal foil shows many drawbacks when used as a pristine electrode without modifications, such as: unlimited volume change, severe formation of Li dendrite, ${ }^{1417}$ low coulombic efficiency, ${ }^{18}$ and most importantly, poor mechanical flexibility for wearable and flexible batteries applications. ${ }^{19}$ Herein, we report a novel Li felt metal anode that can effectively alleviate the aforementioned drawbacks of LIBs and pristine $\mathrm{Li}$ foil anode batteries. Meanwhile, it acquires mechanical flexibility, long term electrochemical stability, and high capacity even under ultra-high current density. This is achieved through the optimum Li metal infiltration into porous carbon felt scaffold. This thermal infiltration technique has been proven recently to grant the batteries extended cycling stability and effectively suppress the dendrite formation at the electrode/electrolyte interface. ${ }^{20,21}$ Which is attributed to: (i) the uniform electrolyte distribution throughout the electrode surface, (ii) reduction of the local current density by increasing the electrode electroactive surface area, and (iii) accommodating the volume expansion and dissipating heat more efficiently during cycling. On the other hand, carbon host scaffolds exhibit distinct advantages over other porous metal scaffolds. This is due to their lower mass density and excellent chemical stability. ${ }^{22-26}$ Additionally, compared to other carbon based materials, carbon felt is characterized by its large area to volume ratio, excellent corrosion resistance, commercial availability, and substantial mechanical flexibility. ${ }^{27,28}$ This inspired us to utilize the carbon felt as a passive scaffold, over which $\mathrm{Li}$ metal is infiltrated to form the Li felt structure. Interestingly, the optimum Li coverage over the carbon felt efficiently sustains the mechanical flexibility of the felt, and provides porosity with enormous electroactive surface area that facilitates ultra-high cycling rates with long-term cycling stability. We 
believe that this work will pave the way to develop 3-dimensional (3D) porous Li metal electrodes, that are not only capable to achieve long term stability under high current densities, but also acquires high flexibility, which is crucial for high energy storage devices to fulfill the needs of the next generation rechargeable batteries.

\section{Results and Discussion}

The Synthesized schematic for the Li felt is shown in Fig. 1a. The carbon felt structure is composed of a uniform cylindrical morphology, over which molten $\mathrm{Li}$ is smoothly infiltrated to form the $\mathrm{Li}$ felt structure. It should be noted that the thermal infiltration conditions should be carefully adjusted, otherwise the molten Li infiltration could completely cover the carbon felt structure and clog all the pores in between the felt. More details about the fabrication method is described in the experimental section. The schematic in Fig. 1b illustrates the ionic deposition mechanism over the Li felt electrode during cycling,

(a)

(b)
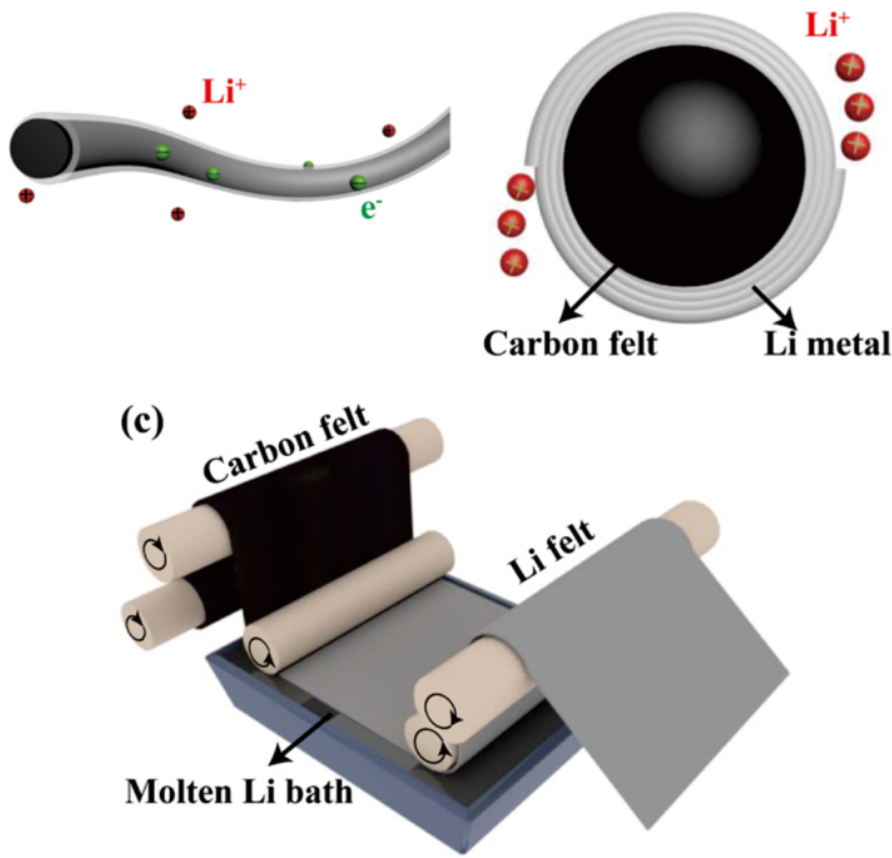

Fig. 1 Schematic diagram for the felt. (a) The Li metal felt formed over the carbon felt scaffold. (b) Cross section schematic for the Li felt that explains $\mathrm{Li}$ ion transport over the surface. (c) Roll-to-roll processing schematic for the Li felt, which can be achieved using the Li melting approach.

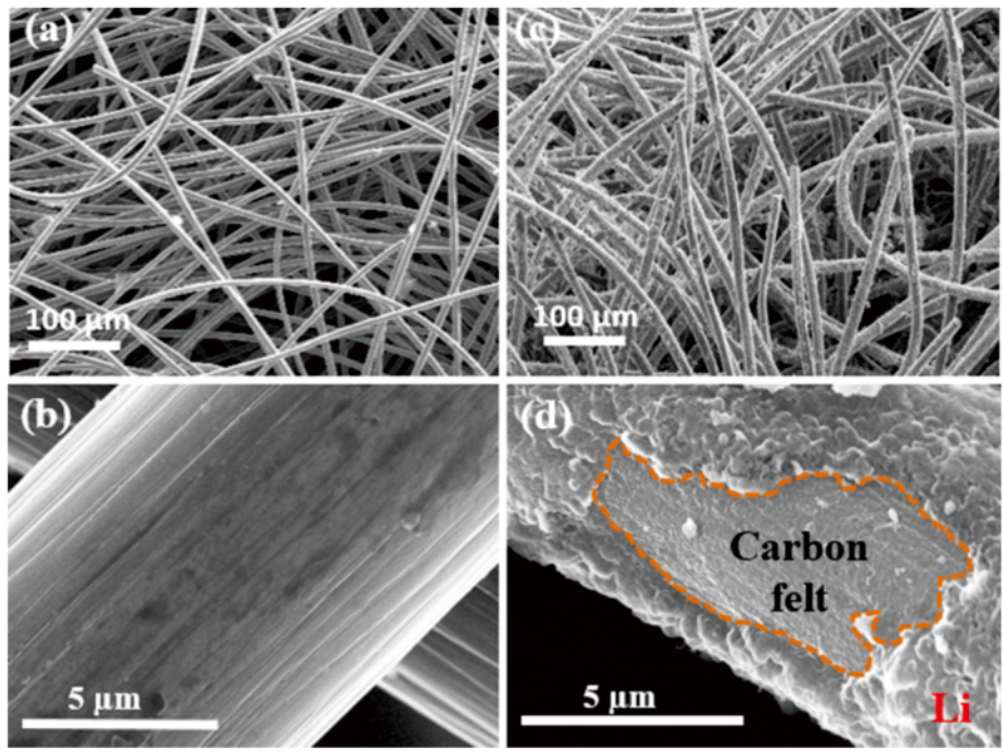

Fig. 2 (a) SEM image for the felt before Li infiltration. (b) The pristine felt under high magnification. (c) SEM image of the felt after Li infiltration. (d) The Li felt under high magnification. 
which takes place homogeneously over the felt surface, owing to the uniform cylindrical morphology, that plays a major role in the outstanding performance for the Li felt. On the practical side, one of the major advantages of the Li thermal infiltration method reported in this study is that it can be easily extended to a large scale, which facilitates the fabrication of Li felt in roll-to-roll set up for industrial implementation as represented in Fig. 1c.

Scanning Electron Microscopy (SEM) images for the carbon felt before and after Li infiltration are presented in Fig. 2. As illustrated in Fig. 2a, the pristine carbon felt acquires highly porous morphology with long extended carbon fibers, which renders the felt enormous internal electrode surface area. A magnified image for a single carbon felt, Fig. $2 \mathrm{~b}$ reveals the uniform cylindrical morphology of the felt, which is substantial to evenly distribute the current density and eliminates the formation of charging current hotspots over the electrode surface. ${ }^{29}$ The optimal Li infiltration over the carbon felt is demonstrated in Fig. 2c. The uniform Li coverage across the carbon felt assists, creating sufficient space for electrolyte diffusion. Further, the large electroactive surface area is maintained after Li infusion, which plays a significant role on both the electrochemical and mechanical properties of the $\mathrm{Li}$ electrode. From the electrochemical side, the extended over-all electroactive surface reduces the local current density and homogeneously distributes the electrolyte during lithiation/delithiation process. Simultaneously, the porous structure limits the volume expansion by confining $\mathrm{Li}$ over a well-defined one-dimensional structure, which efficiently limits the $\mathrm{Li}$ electrode volume expansion. ${ }^{20}$
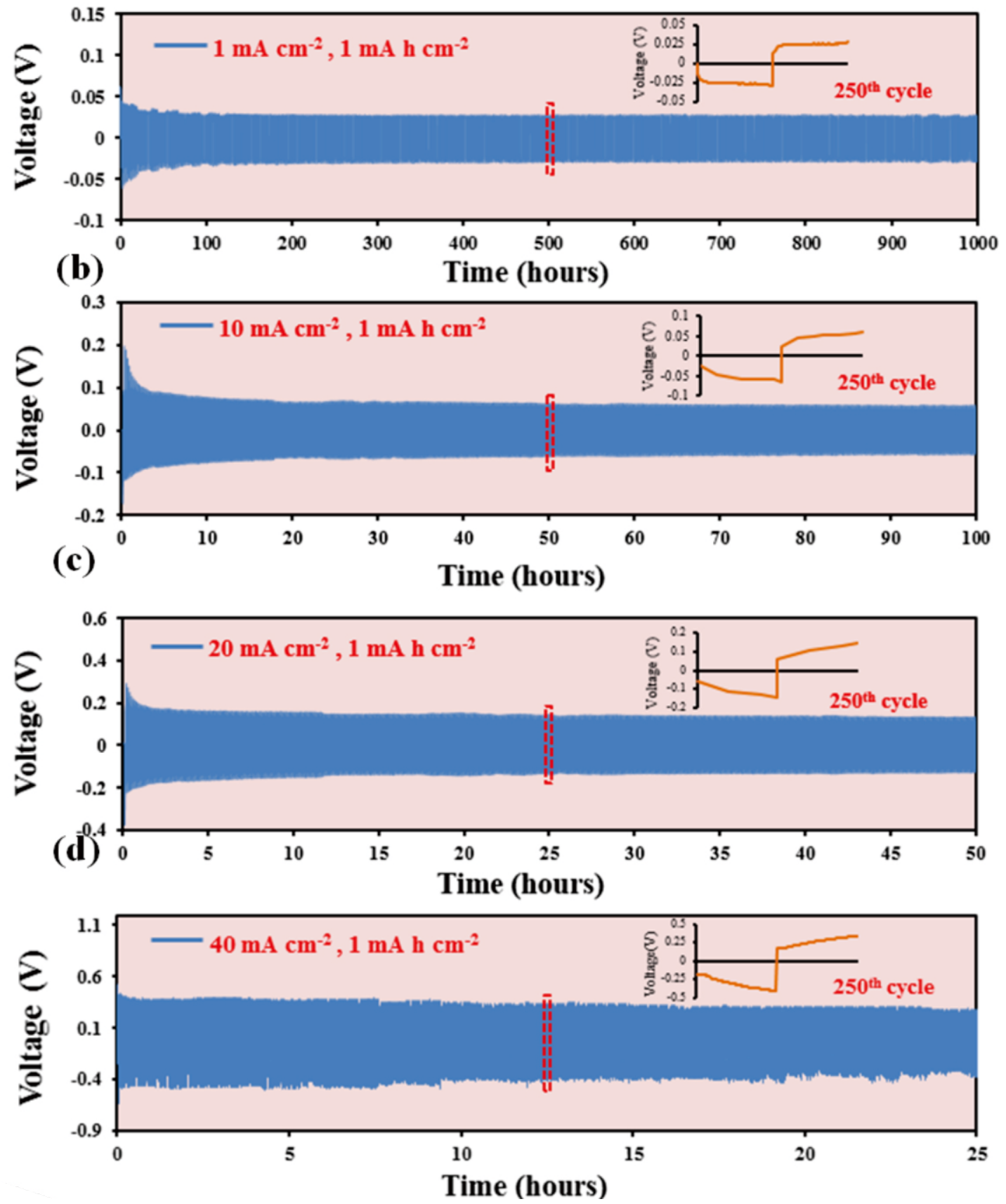

Fig. 3 Cycling stability of Li felt symmetric cell at $1 \mathrm{~mA} \mathrm{~h} \mathrm{~cm}^{-2}$ for 500 cycles at (a) $1 \mathrm{~mA} \mathrm{~cm}^{-2}$, (b) $10 \mathrm{~mA} \mathrm{~cm}$, (c) $20 \mathrm{~mA} \mathrm{~cm}$, and (d) $40 \mathrm{~mA} \mathrm{~cm}$. The inset figures shows the platting/stripping curve for the $250^{\text {th }}$ cycle at each cycling rate. 
From the mechanical characteristics point of view, Li metal perfectly follows the morphology of the carbon felt, forming a shell-like shape over the felt surface with $\sim 700 \mathrm{~nm}$ wall thickness, which retains the mechanical flexibility of the pristine felt and provides more robustness to the electrode structure. Fig. $2 \mathrm{~d}$ reveals a magnified image for the $\mathrm{Li}$ felt. The felt preserves a uniform cylindrical morphology, which assures uniform ionic distribution across the surface and provides more stable solid electrolyte interface (SEI) layer because of eliminating large charging/discharging current hotspots during lithiation/delithiation. Snapshots for the Li felt structure is shown in Fig. S1 in the supporting information. Fig. S1a represents the $\mathrm{Li}$ felt surface, which reveals insignificant change in the felt morphology after Li infiltration. As mentioned earlier, it is crucial to grant the diffusion of the electrolyte through the electrode and to sustain the felt flexibility. The flexibility of the Li felt can be also observed from Fig. S1b, which further facilitates the roll-to-roll fabrication of the Li felt on industrial scale.

In order to reveal the outstanding electrochemical stability of the Li felt electrode, Galvanostatic cycling is performed using symmetric coin cells with two identical Li-felt samples. The test was conducted at different current densities: $1 \mathrm{~mA} \mathrm{~cm}{ }^{-2}, 5 \mathrm{~mA} \mathrm{~cm}^{-2}, 10 \mathrm{~mA} \mathrm{~cm}^{-2}$, and up to $40 \mathrm{~mA} \mathrm{~cm}^{-2}$ for 500 cycles, all under fixed charging capacity of $1 \mathrm{~mA}$ $\mathrm{h} \mathrm{cm}^{-2}$. As observed from Fig. 3a, the felt shows high stability under low current density of $1 \mathrm{~mA} \mathrm{~cm}^{-2}$. This stability is well maintained at $10 \mathrm{~mA}$ $\mathrm{cm}^{-2}$, as shown in Fig. 3b, with small overpotential increase from $0.03 \mathrm{~V}$ to $0.07 \mathrm{~V}$ as a result of the increased ohmic resistance with higher current densities. Interestingly, upon applying a higher current density of $20 \mathrm{~mA} \mathrm{~cm}^{-2}$ (Fig. 3c), the cycling not only shows high stability with insignificant hysteresis, but also acquires small overpotential of about $0.15 \mathrm{~V}$ even after 500 cycles. At ultra-high current density of $40 \mathrm{~mA}$ $\mathrm{cm}^{-2}$ (Fig. 3d), the structure shows limited voltage hysteresis during cycling, however the sample still maintains significant stability over the 500 cycles. From the inset figures, we can also observe that the Li felt shows quite flat voltage plateaus without any obvious overpotential "bump" during the charging and discharging processes under the different applied current densities, which indicates low nucleation and stripping energy barriers. This is attributed to the high electroactive surface area provided by the felt structure that enables homogeneous 3D Li Platting/stripping, enabling low-energy barrier during the cycling process. ${ }^{30}$ Meanwhile, the large electroactive surface area between the felt and the electrolyte decreases the overall current density throughout the surface, and allows the electrode to sustain stability even under 40 $\mathrm{mA} \mathrm{cm}{ }^{-2}$ ultrahigh cycling current. On the other hand, the conductivity of the carbon felt scaffold underneath the Li layer supports high conducting pathways for electrons transport. Hereby decreases the interfacial resistance, which is evident from the low overpotential obtained during the symmetric cell test. Additionally, the cylindrical shape of the felt provides uniform distribution for Li ions over the electrode surface and eliminates the formation of charging hotspots areas, which facilitates the evolution of stable SEI layer and suppresses the dendrite formation, which is confirmed from the limited hysteresis and long term stability during cycling.

To further investigate the interfacial resistance and the stability of the SEI layer, electrochemical impedance spectroscopy (EIS) is performed for both, Li felt and Li metal foil symmetric cells. It should be noted that the high-frequency semicircle represents the interfacial resistance for the migration of $\mathrm{Li}$ ions at the electrolyte/electrode interface, whereas the intersection of the semicircle with the $\operatorname{Re}(Z)$ axis represents the cell overall ohmic resistance. Fig. 4a shows the Nyquist plots before cycling, as evident from the figure, the Li foil electrode shows interfacial resistance of $405.6 \Omega$, this high interfacial resistance is ascribed to the native oxide layer formed on the surface of $\mathrm{Li}$ foil electrode. ${ }^{20}$ On the other hand, the Li felt electrode reveals significant lower interfacial resistance $(182.7 \Omega)$, which indicates more uniform electrolyte distribution throughout the electrode surface, and confirms the formation of more stable SEI layer with better charge transport across the interface. Fig. $4 \mathrm{~b}$ represents the Nyquist plots after 25 cycles at $20 \mathrm{~mA} \mathrm{~cm}$, obviously, the $\mathrm{Li}$ foil interfacial resistance is reduced down to $112.6 \Omega$ as a result of the oxide layer removal after cycling. On the other hand, the interfacial resistance for the Li felt electrodes is magnificently reduced to $20 \Omega$, which is attributed to the formation of more stable SEI layer and better kinetics for the charge transport across the interface during cycling. These results further illustrate the outstanding stability and low overpotential of the felt electrodes with long-term cycling, as indicated previously in Fig. 3. Moreover, it confirms the capability of the $\mathrm{Li}$ felt to induce the formation of extremely stable SEI layer, and to afford better charge transport kinetics during cycling, even under ultra-high charging/discharging current densities. The long term cycling stability is ascribed to the uniform cylindrical morphology of the Li layers, as well as the confined and direct pathways for electrons transport provided by the felt.

The main deficiency caused by the large electrode interfacial resistance, is the limitation of the battery specific power, where large portion of the power is dissipated in the form of localized heat over the electrode surface during charging/discharging. Under high current loads, the heat evolution becomes predominant, and signifucantly affects the energy efficiency and the overall performance of the battery. ${ }^{31}$ Apparently, in order to operate the Li felt under high current densities, prescience estimation of the interfacial resistance effects and the power dissipation over the felt electrode surface is critically required. For this, we demonstrated a symmetric cell Multiphysics simulation model for investigating the electrochemical stripping/platting process, including both, $\mathrm{Li}$ felt and $\mathrm{Li}$ foil symmetric cells under similar applied potential. The model mimics the electrochemical reactions at the electrodes' surface during lithiation/delithiation as well as the surface deformations that occur during the process, which gives deep insights regarding the Li growth mechanism, as well as the effect of the surface morphology on the electrode stability. More details about the simulation model including the governing equations and the parameters used in this study, can be found in the supporting information.

The normalized power dissipated across the symmetric cell for the $\mathrm{Li}$ felt and its $\mathrm{Li}$ foil cell counterpart is represented in Fig. $4 \mathrm{c}$, d, respectively. Distinctly, the power dissipated from the felt surface is homogeneously distributed throughout the felt surface compared to its pristine $\mathrm{Li}$ foil electrode counterpart. This is mainly attributed to the higher interfacial resistance of the $\mathrm{Li}$ foil during the lithiation/delithiation process as confirmed form the EIS test. Moreover, it can be noticed that the sharp edges at the foil surface acquires higher power dissipation values, at which the localized heating is difficult to dissipate and the current flow is large, which affects the battery performance and long cycle life. ${ }^{32}$ Contrarily, the felt shows more efficient power dissipation, which minimizes the localized heating across the electrode surface. These results interpret the outstanding stability of the felt structure at ultra-high current density during the cycling test. Where the power dissipation is optimized so that the surface stability significantly enhanced. Moreover, the results are consistent with the EIS experimental results, and confirms the preference of the Li felt over the bulk Li foil electrodes.

From the previous discussion, it can be concluded that the interfacial resistance plays critical role in determining the cell stability and performance. The interfacial resistance, and hence, the electrochemical stability are strongly related to the ion distribution over the electrode surface. The more uniform ionic charge distribution, the 
(a)

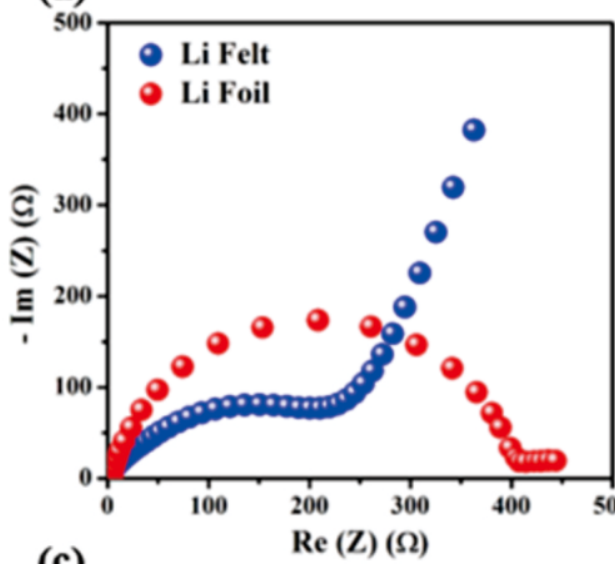

(c)

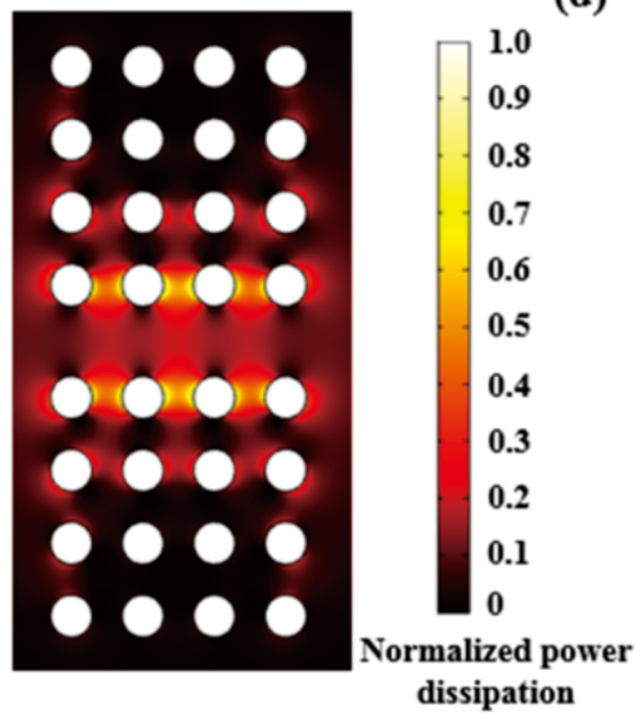

(b)

(d)
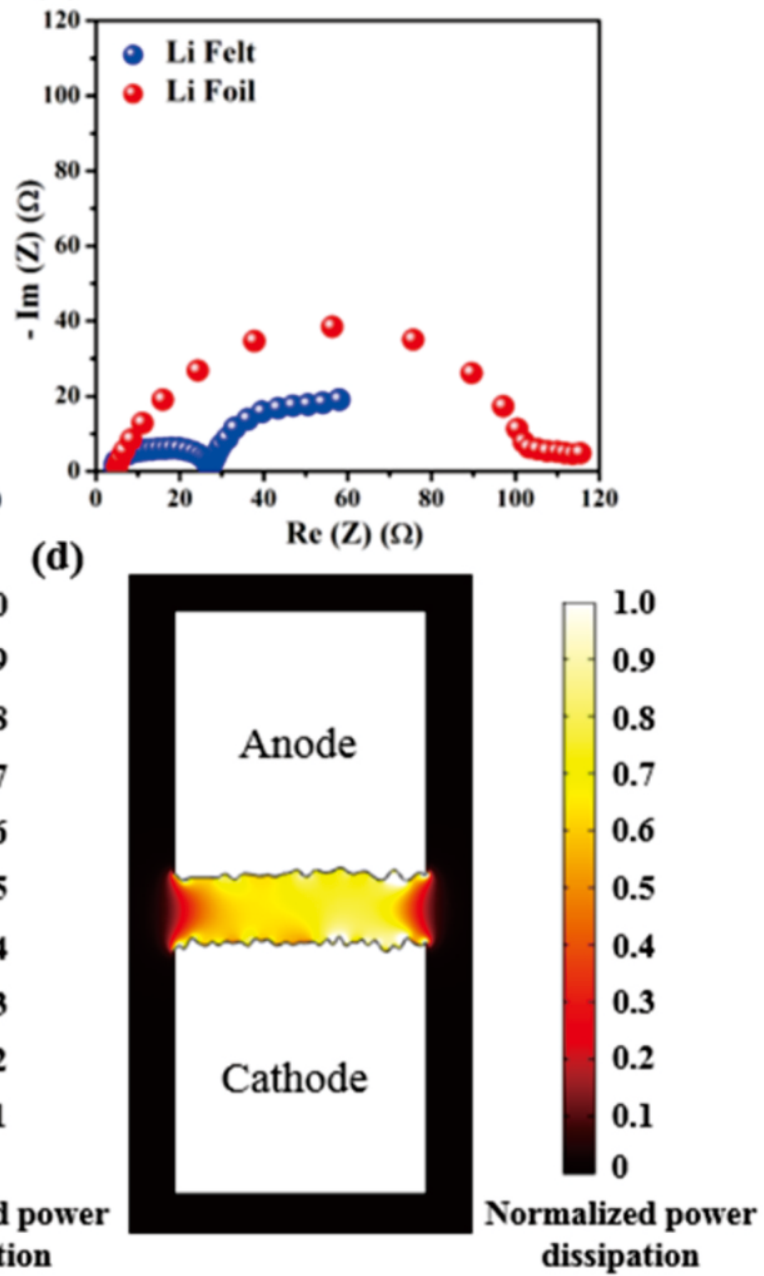

Fig. 4 Electrochemical impedance spectroscopy (EIS) for the Li felt (blue), and the Li foil (red) before cycling (a) and after 20 cycles (b). (c) Normalized power dissipation over the Li felt electrode. (d) Normalized power dissipation over the Li foil electrode.

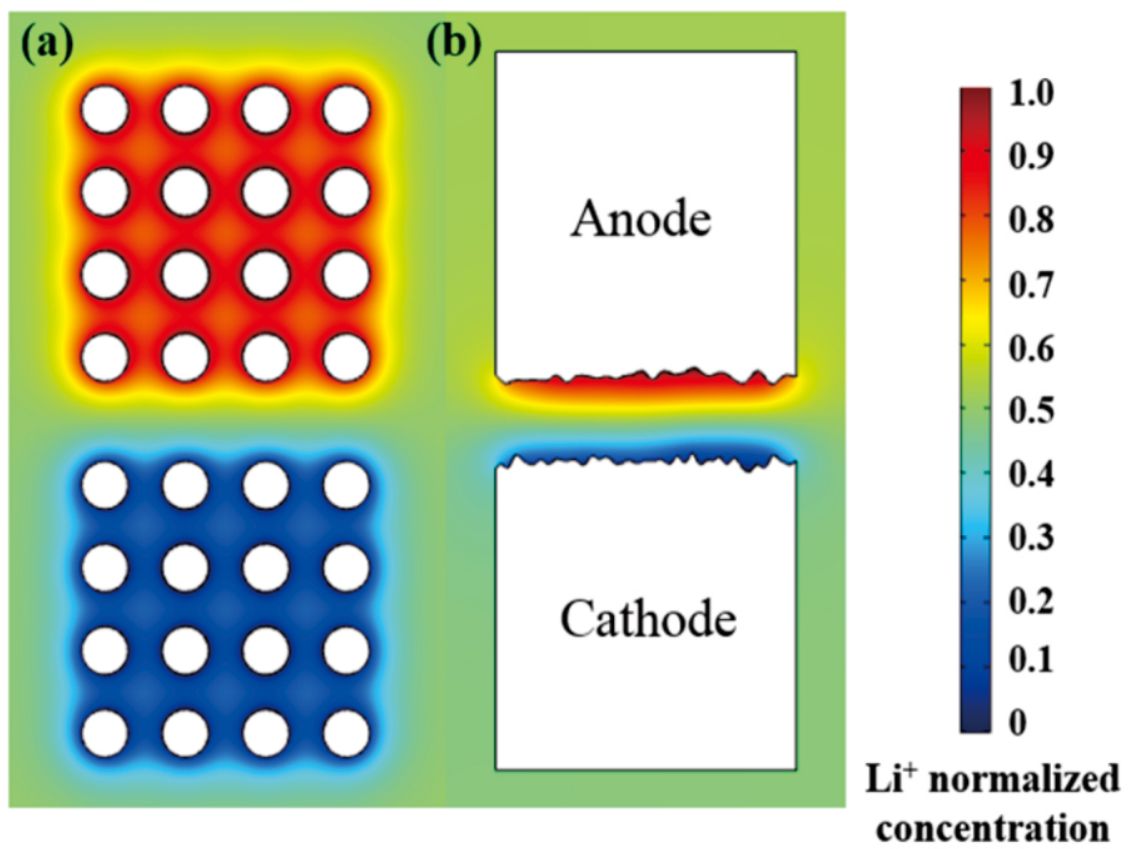

Fig. 5 Normalized Li ionic distribution throughout the electrodes in the symmetric cell design. (a) Li felt symmetric cell. (b) Li foil symmetric cell. 
more stable SEI layer formation. Consequently, we investigated the $\mathrm{Li}$ ionic charge distribution throughout the cell during the lithiation/delithiation process. Fig. 5 represents the normalized $\mathrm{Li}^{+}$ concentration across the symmetric cell for the $\mathrm{Li}$ felt, as well as its $\mathrm{Li}$ foil cell counterpart. As expected, the concentration of $\mathrm{Li}$ ions is maximum at the anode side during discharging, as a result of the $\mathrm{Li}$ metal dissolution process from the electrode surface. On the other hand, the $\mathrm{Li}$ ion concentration is minimum at the cathode side, at which the $\mathrm{Li}$ deposition takes place. From Fig. 5a, we can clearly observe the high uniformity of the $\mathrm{Li}$ ion species distribution at both the anode and cathode electrodes. This indicates that the lithiation/delithiation process takes place homogeneously throughout the felt structure. This ordered process grants the formation of extremely stable and robust SEI layer at the electrode surface with uniform thickness. As a result, the cracking of the SEI layer during cycling is greatly suppressed and the interfacial resistance is significantly reduced. On the other hand, the $\mathrm{Li}$ ion concentration over the Li foil surface (Fig. 5b) is randomly distributed either at the anode or the cathode side. Moreover, the electrode surface exhibits different ion concentration gradient at different areas, particularly, at the regions with higher surface roughness. This severely affects the stability of the SEI layer formed during cycling, as well as increasing the interfacial resistance of the electrodes. These results are also in agreement with the electrochemical cycling and EIS observations, and further explain the fundamentals behind the outstanding cycling performance of the porous $\mathrm{Li}$ felt compared to its Li foil counterpart.

In order to get more insights about the surface morphology for the felt as well as the Li foil electrodes before and after cycling, SEM measurements were conducted after 50 cycles at $10 \mathrm{~mA} \mathrm{~cm}{ }^{-2}$ current density, and $1 \mathrm{~mA} \mathrm{~h} \mathrm{~cm}$ charging capacity. Fig. 6a-c represents the surface morphologies of both electrodes before cycling. Fig. 6a, b represents lower and higher magnification of the carbon felt, whereas Fig. $6 \mathrm{c}$ represents the pristine $\mathrm{Li}$ foil surface, which acquires random morphology with limited electroactive surface area for electrolyte diffusion. This enhances the formation of hotspot currents that causes SEI layer instability that finally breaks and triggers Li dendrites to shoot up from the electrode surface. Contrarily, the felt eliminates the aforementioned problems through its uniform and porous 3D structure. This becomes more evident by investigating the structure morphologies after stripping (Fig. 6d-f), and platting (Fig. 6g-i) for both electrodes. From the felt stripping images (Fig. 6d, e), we can observe that $\mathrm{Li}$ stripping mechanism strongly affected by the electrode morphology. The cylindrical morphology distributes the electrostatic charge symmetrically over the electrode surface, which facilitates the stripping of $\mathrm{Li}$ metal from the outer surface layers, followed by sequential $\mathrm{Li}$ stripping from the inner layers. This systematic pealing process for $\mathrm{Li}$ metal from the felt surface, plays critical role in stabilizing the SEI layer and suppressing the dendrite formation at high current densities. On the
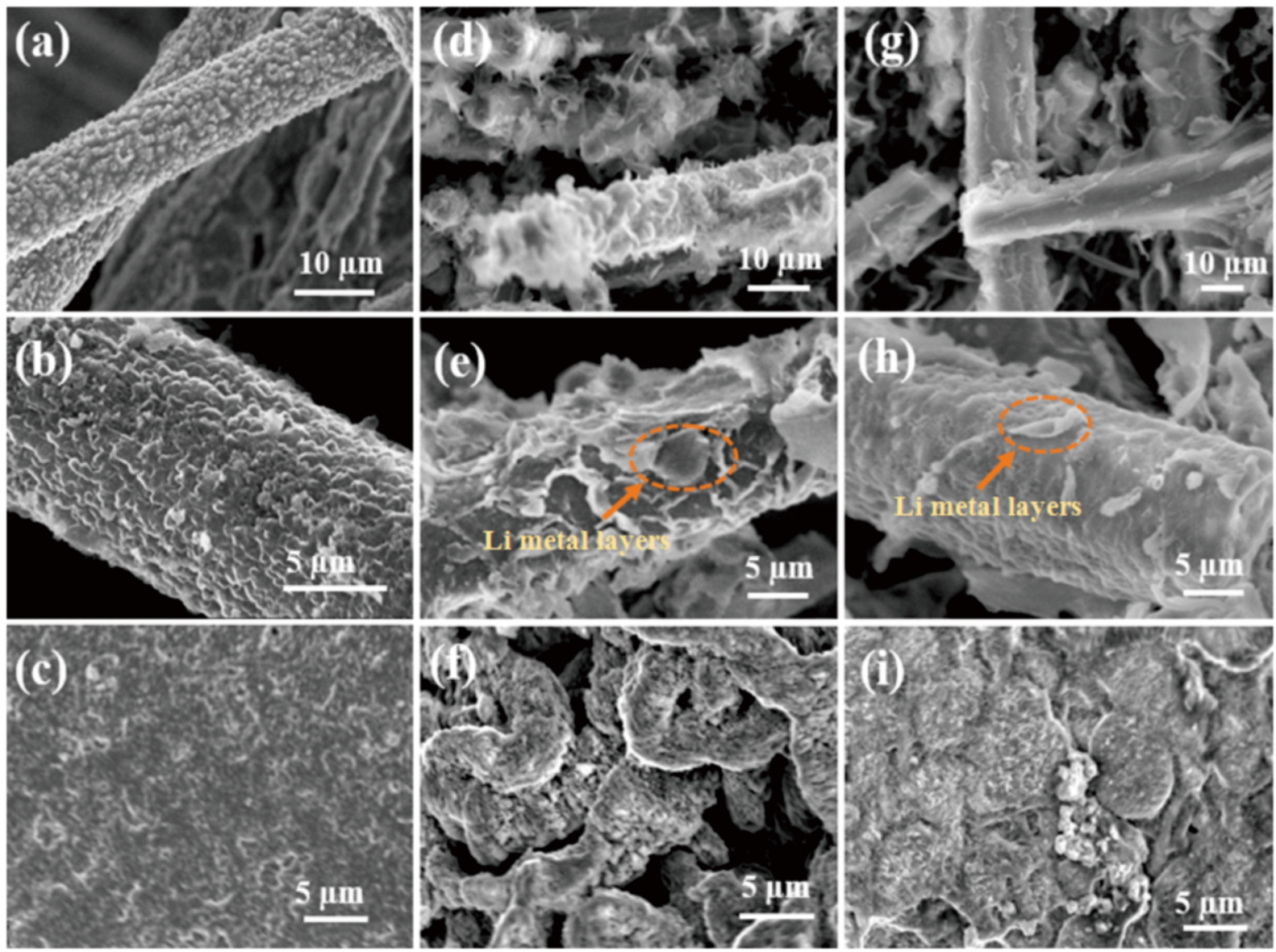

Fig. 6 SEM images for the surface morphology of the Li felt and Li foil structures before and after 25 cycles. (a) and (b), Li felt before cycling at different magnification. (c) Li foil before cycling. (d) and (e), Li felt stripping at different magnification. (f) Li foil stripping. (g) and (h) Li felt platting at different magnification. (i) Li foil platting. 
other hand, Li foil shows severe dendrite formation after 25 cycles at 10 $\mathrm{mA} \mathrm{cm} \mathrm{cm}^{-2}$ as obvious in Fig. 6f. On the platting side, Fig. $6 \mathrm{~g}$, h reveal uniform Li platting over the felt surface, and from the high magnification image (Fig. 5h), it can be observed that Li surface is more uniform without noticeable dendrite formation. Contrarily, The $\mathrm{Li}$ foil platting is shown in Fig. 6i, where the foil surface shows severe dendrite formation with marginal surface roughness.

To elucidate the stripping/platting mechanism for the Li felt and to investigate the reason behind its outstanding stability compared to its pristine Li foil counterpart, the normalized current density across the cell is simulated. Fig. 7 represents the electrolyte current density through the coin cell after cycling, upon applying a stripping and platting overpotential of $10 \mathrm{mV}$. From Fig. 7a, it can be observed that the Li foil surface acquires random charge density distribution, which is mainly evolved from the surface roughness and the non-uniform morphology across the electrode surface. Also, we can observe the presence of hotspot areas with higher current densities at the sharp edges over the electrode surface, at which the lithiation/delithiation rate varies significantly, that plays the main role in further surface deformations during cycling, which leads to SEI cracking and dendrite formation as stated earlier. It should be mentioned that upon small increase in the applied over potential the current charge density becomes more random across the surface with much higher values at the sharp edges of the foil. These interesting results, reveals the reason behind the instability and short circuit problems that occurs during $\mathrm{Li}$ metal foil cycling, and further explains the effect of surface morphology on the stability of the Li foil. Animation movie S2 also reveals the time evolution for the Li foil stripping/platting process, eventually, random $\mathrm{Li}$ growth is observed during platting, with uncontrolled volume change that deteriorates the surface stability, and can easily break the SEI layer formed on the surface, consuming large amount of the electrolyte Li salt concentration. On the other hand, Fig. $7 \mathrm{~b}$ shows the Li felt cross section subjected to the same applied potential. Significant reduction in the overall current density is clearly observed, that is attributed to the large electroactive surface area acquired by the felt compared to its Li foil counterpart. Further, highly uniform charge distribution is observed across the felt surface, which regulates the lithiation/delithiation process throughout the electrode structure. This explains the reason behind the outstanding stability observed during the Li felt cycling test even at ultra-high current density of $40 \mathrm{~mA} \mathrm{~cm}$. It should be noted that the boundary felt layers of the electrodes has relatively non-uniform current density distribution. Which is mainly related to the electrostatic charge variations at the outer electrode surface between the positive and negative electrodes, however, this effect is eliminated at the core layers, and more uniform charge distribution can be observed around the core felt surface layers. These results interprets and agrees with the SEM results mentioned above, where smooth surface was clearly observed after $\mathrm{Li}$ platting as a result of the regulated $\mathrm{Li}$ ionic flux over the felt. Additionally, the animation movie S3, shows the stripping/platting process through time, where we can observe the consistent morphology change of the felt structure, in contrast to the random geometric change of the $\mathrm{Li}$ foil surface as a result of the random lithiation/delithiation process across the surface. These results also support our claim that the stripping/platting of Li ions over the felt structure takes place layer by layer based on the charge distribution. Which further explains the formation of plate-like structure that is observed in the SEM figures.

To evaluate the Li felt full cell performance, two full coin cell sets was assembled, with $\mathrm{LiFePO}_{4}$ as cathode, and either $\mathrm{Li}$ felt or $\mathrm{Li}$ foil as anode. As shown in Fig. 8a, the $\mathrm{Li}$ felt shows outstanding rate performance compared to its pristine Li foil counterpart. Particularly, at high charging rates of $10 \mathrm{C}$ and $20 \mathrm{C}$, the felt exhibits specific capacity of $\sim 70 \mathrm{~mA} \mathrm{~h} \mathrm{~g}^{-1}$ and $43 \mathrm{~mA} \mathrm{~h} \mathrm{~g}^{-1}$, respectively, compared to $\mathrm{Li}$ foil that shows much lower capacity of $\sim 10 \mathrm{~mA} \mathrm{~h} \mathrm{~g}^{-1}$ and $5 \mathrm{~mA} \mathrm{~h} \mathrm{~g}^{-1}$ at $10 \mathrm{C}$ and $20 \mathrm{C}$, respectively. Which is related to the high interfacial resistance of the $\mathrm{Li}$ foil interface that has great impact in deteriorating the cell performance as discussed earlier. However, Li felt acquires lower interfacial resistance arising from better charge mobility and uniform lithiation/delithiation. Fig. 8b, shows the long term stability test over 150 discharging cycles at $1 \mathrm{C}$ rate. The Coulombic efficiency was calculated as the charge ratio of the $\mathrm{Li}$ dissolution to that of the $\mathrm{Li}$ deposition at the anodic side. Obviously, the Li felt shows superior stability with about $99.8 \%$ Coulombic efficiency during cycling.
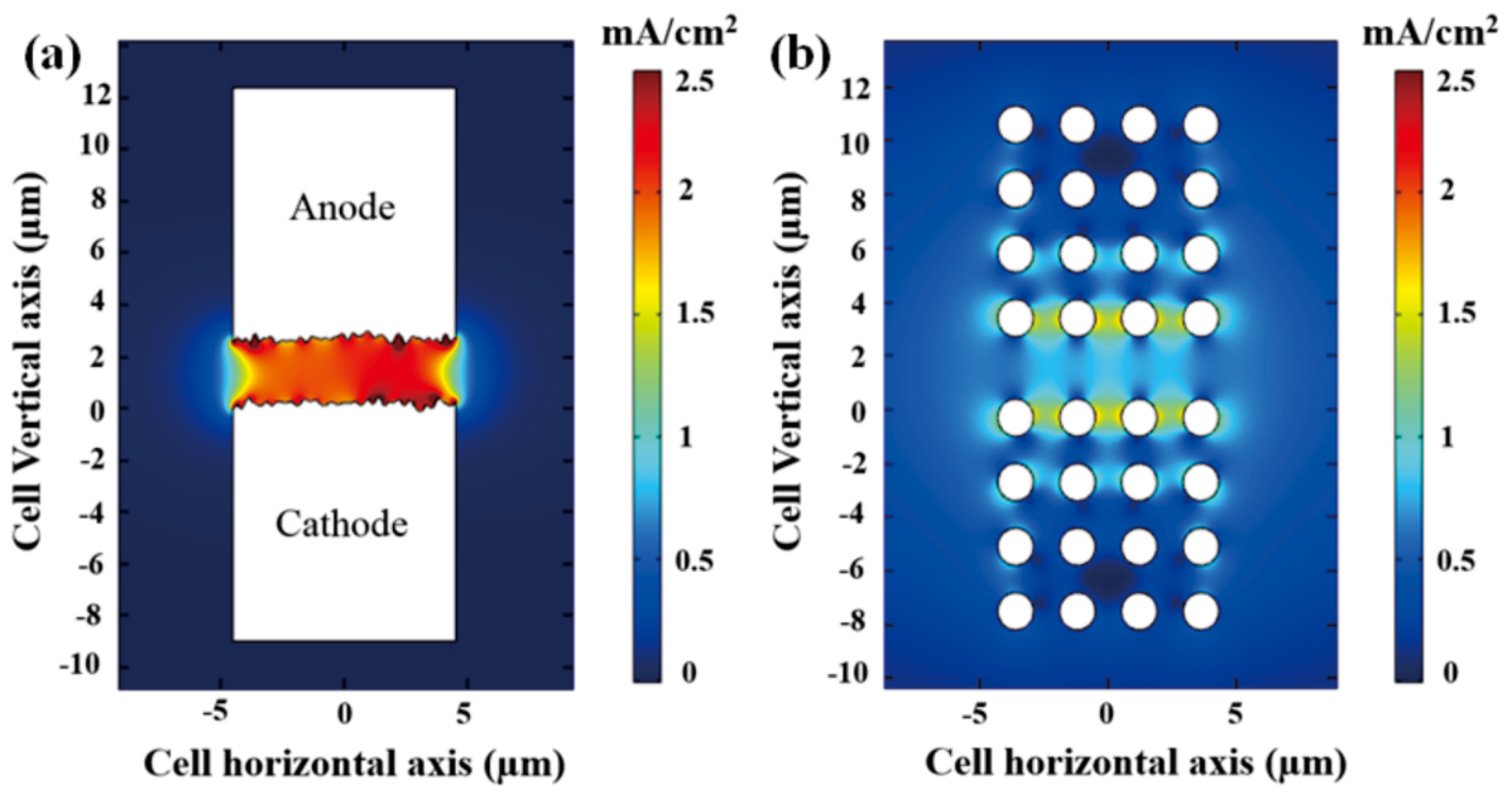

Fig. 7 Simulation results for the electrolyte current charge density distribution across the coin cell. (a) Li foil symmetric cell. (b) Li felt symmetric cell. 
However, the Li foil cells shows lower efficiency of about $98.1 \%$ with significant capacity hysteresis. Moreover, The Li felt retains $99.2 \%$ of its initial capacity after 150 cycles, whereas the Li foil electrode capacity retention was only $\sim 94 \%$ of its initial capacity. This declining in the capacity retention is related to the uncontrolled volume change during cycling, that breaks the SEI layer and consumes more electrolyte $\mathrm{Li}$ salt to rebuild the broken SEI regions, which finally leads to severe dendrite formation and decaying in the cell performance. On the other hand, the Li felt acquires higher stability with large capacity retention as a result of the stable SEI layer formed at the cylindrical felt surface, which agrees with the simulation results, and further confirms the effect of the surface morphology on the overall cell performance. Fig. 8c, d shows the voltage profile for both anodes at $0.5 \mathrm{C}$ and $5 \mathrm{C}$, respectively. In Fig. 8c, an obvious flat voltage plateau is observed for the Li felt sample compared to its Li foil counterpart. At higher charging rates (Fig. 8d), the Li felt sample still shows better voltage plateau with lower voltage overpotential at large specific capacity. This also well agrees with the symmetric coin cell test superior performance, and further declares the extended stability of the Li felt samples compared to their Li foil counterparts.

\section{Conclusion}

In this work, we have introduced a novel structure for Li metal anodes in the form of Li felt. The structure revealed unprecedented stability at

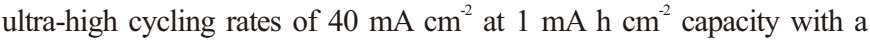
significantly small voltage hysteresis over 500 cycles. The SEM images revealed that the $\mathrm{Li}$ felt maintains high porosity even after $\mathrm{Li}$ infiltration, which provides extremely large electroactive surface area that allows more uniform electrolyte distribution, reduced local current density, and homogeneous Li deposition/dissolution. The EIS results showed that the $\mathrm{Li}$ felt structure acquires lower interfacial resistance than its Li foil counterpart, which became more evident with cycling. The SEM images for the Li felt stripping/plating revealed that the felt structure can effectively suppress the dendrite formation, in contrast to the $\mathrm{Li}$ foil structure that showed severe dendrite formation across the surface. Furthermore, the surface revealed a $\mathrm{Li}$ plate-like structure formed during stripping/plating process, which suggests that the lithiation/delithiation process takes place over the felt surface. These results are confirmed by the finite element simulation models, which illustrated in more detail the effect of surface morphology on the outstanding stability of the felt structure compared to its pristine Li foil counterpart. Moreover, we performed a full cell test to investigate the $\mathrm{Li}$ felt performance, with $\mathrm{Li}$ felt as anode and $\mathrm{LiFePO}_{4}$ as cathode. The felt showed 7 times higher capacity than Li foil at $10 \mathrm{C}$, and 9 times higher capacity at $20 \mathrm{C}$. The long term stability test at $1 \mathrm{C}$ revealed the significant stability of the Li felt compared to its Li foil counterpart, with capacity retentions of $\sim 99.2 \%$ and $\sim 94 \%$ respectively, after 150 cycles. Meanwhile, the coulombic efficiency of the felt was $\sim 99.8 \%$ compared to Li foil with $\sim 98.1 \%$ efficiency over the cycling period. At the end, we believe that this novel $\mathrm{Li}$ felt structure with its superior electrochemical stability and mechanical flexibility, combined with its simple and low cost fabrication method reported in this work, will pave new avenue for enhancing the performance of Li metal anodes, and release limitations that hinders its practical utilization in rechargeable

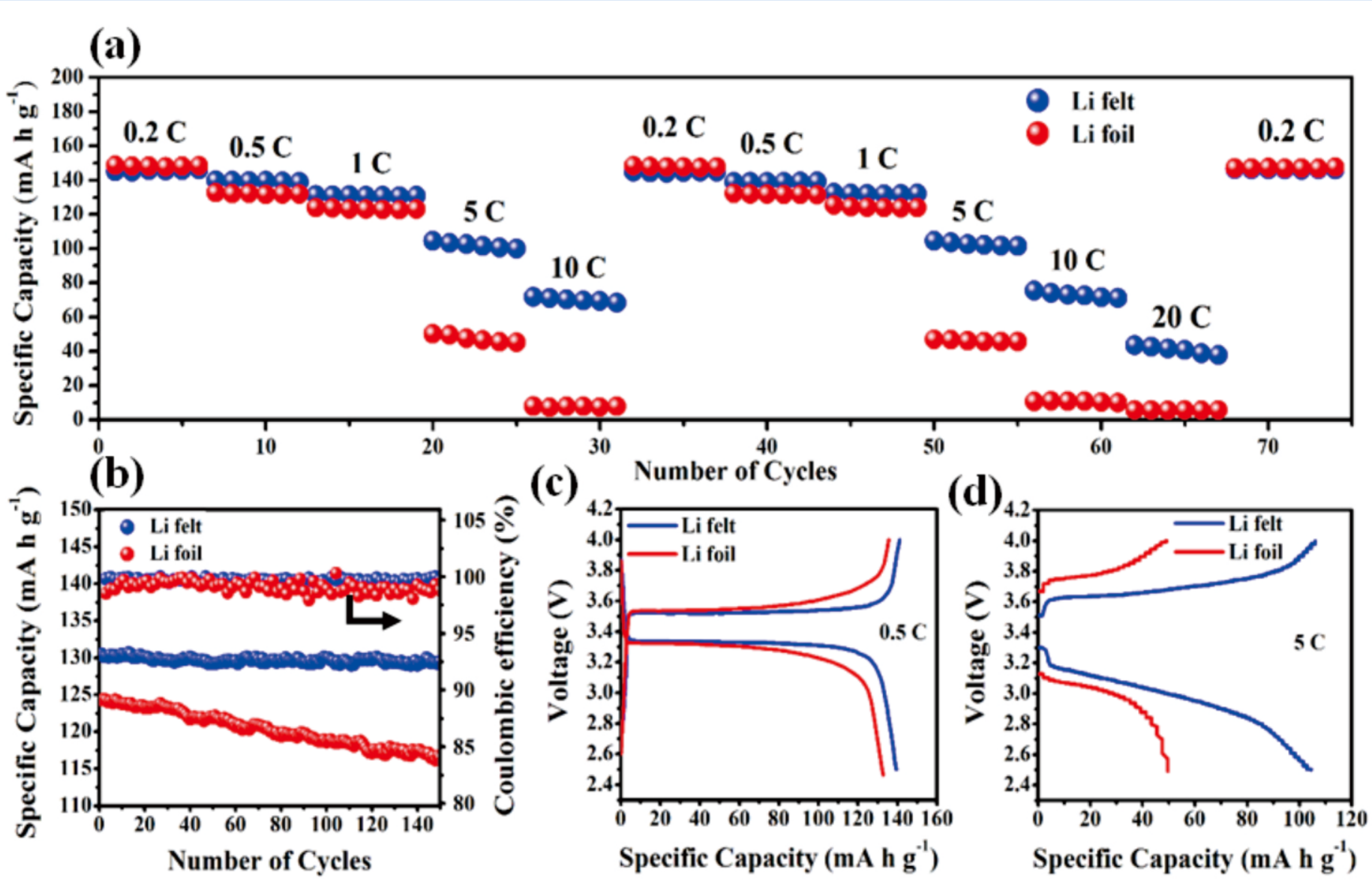

Fig. 8 Electrochemical performance for $\mathrm{LiFePO}_{4} / \mathrm{Li}$ felt (blue) and $\mathrm{LiFePO}_{4} / \mathrm{Li}$ foil (red) full cell test. (a) Rate capability under different rates from $0.2 \mathrm{C}$ to $20 \mathrm{C}$. (b) Long term cyclic stability at $1 \mathrm{C}$. (c,d) Voltage charging/discharging profiles at $0.5 \mathrm{C}$ and $5 \mathrm{C}$, respectively. 
batteries.

\section{Experimental Section}

Preparation of the Li felt electrode:

Commercial carbon field was firstly rinsed in ethanol, then soaked in $0.7 \mathrm{M}$ zinc nitrate $\left(\mathrm{Zn}\left(\mathrm{NO}_{3}\right)_{2}\right)$ in ethanol, the structure was then annealed at $400{ }^{\circ} \mathrm{C}$ for 5 minutes. The felt was then transferred into argon filled glove box for Li infilteration. Fresh Li ribbons (99.9\%, Sigma Aldrich) was carefully scraped to completely remove oxide layers formed on the surface. The pure shiney Li was then melted at elevated temperature of $450{ }^{\circ} \mathrm{C}$. Meanwhile, The felt was also sintered at the same tempertaure for 10 mins, this to assure that the molten $\mathrm{Li}$ will be infilterated smoothly through the foam structure without aglomorations and intimately cover the felt fibers' surface.

\section{Electrochemical Characterizations:}

For the galvanostatic symmetric cell test, The electrodes were assembled into a symmetric cell configuration with (2025-MTI) coin cell type. In this setup, the electrodes used for this test were Li metal, and $\mathrm{Li}$ felt symmetric electrode pairs. For the full cell test, Li felt was used as anode, whereas $\mathrm{LiFePO}_{4}$ was used as cathode electrode. In the counter full cell, Li coin metal (MTI) was used as anode instead, and $\mathrm{LiFePO}_{4}$ was used as cathode. The active cathode material was prepared with polyvinylidine fluoride (PVDF, MTI) and carbon black with a ratio of 8:1:1 in N-Methyl-2-pyrrolidone (NMP) solvent, indicated by MTI. The electrolyte used in all tests was $1 \mathrm{M} \mathrm{Li}$ bis (trifluoromethanesulphonyl) imide (LiTFSI) in 1:1 v/v 1,3-dioxolane (DOL)/1,2-dimethoxyethane (DME) with 2 wt.\% Li nitrate. Celgard $2325(25 \mu \mathrm{m})$ was used as separator. For the measurements, LANDTH 8-channel tester was used for the Galvanostatic cycling tests, while Biologic VMP3 potentiostat was used to conduct the electrochemical impedance spectroscopy test.

\section{References}

1. M. Stoppa and A. Chiolerio, Sensors, 2014, 14, 11957.

2. D. H. Kim, N. Lu, R. Ma, Y. S. Kim, R. H. Kim, S. Wang, J. Wu, S. M. Won, H. Tao and A. Islam, Science, 2011, 333, 838.

3. W. Zeng, L. Shu, Q. Li, S. Chen, F. Wang and X. M. Tao, Adv. Mater., 2014, 26, 5310 .

4. K. Jost, D. Stenger, C. R. Perez, J. K. McDonough, K. Lian, Y. Gogotsi and G. Dion, Energy Environ. Sci., 2013, 6, 2698.

5. S. W. Kim, J. H. Yun, B. Son, Y. G. Lee, K. M. Kim, Y. M. Lee and K. Y. Cho, Adv. Mater., 2014, 26, 2977.

6. A. E. Ostfeld, A. M. Gaikwad, Y. Khan and A. C. Arias, Sci. Rep-UK, 2016, 6, 26122.

7. G. Zhou, F. Li and H. M. Cheng, Energy Environ. Sci., 2014, 7, 1307.

8. B. Scrosati, J. Hassoun and Y. K. Sun, Energy Environ. Sci., 2011, 4, 3287.
9. N. S. Choi, Z. Chen, S. A. Freunberger, X. Ji, Y. K. Sun, K. Amine, G. Yushin, L. F. Nazar, J. Cho and P. G. Bruce, Angew. Chem. Int. Edit., 2012, 51, 9994.

10. K. Liu, B. Kong, W. Liu, Y. Sun, M. S. Song, J. Chen, Y. Liu, D. Lin, A. Pei and Y. Cui, Joule, 2018, 2, 1857.

11. J. S. Kim, D. Ko, D. J. Yoo, D. S. Jung, C. T. Yavuz, N. I. Kim, I. S. Choi, J. Y. Song and J. W. Choi, Nano lett., 2015, 15, 2350.

12. W. Xu, J. Wang, F. Ding, X. Chen, E. Nasybulin, Y. Zhang and J. G. Zhang, Energy Environ. Sci., 2014, 7, 513.

13. H. Kim, G. Jeong, Y. U. Kim, J. H. Kim, C. M. Park and H. J. Sohn, Chem. Soc. Rev., 2013, 42, 9011.

14. C. P. Yang, Y. X. Yin, S. F. Zhang, N. W. Li and Y. G. Guo, Nat. Commun., 2015, 6, 8058 .

15. Z. Li, J. Huang, B. Y. Liaw, V. Metzler and J. Zhang, J. Power Sources, 2014, 254, 168.

16. J. Zhao, G. Zhou, K. Yan, J. Xie, Y. Li, L. Liao, Y. Jin, K. Liu, P. C. Hsu and J. Wang, Nat. Nanotechnol., 2017, 12, 993.

17. P. Bai, J. Li, F. R. Brushett and M. Z. Bazant, Energy Environ. Sci., 2016, 9, 3221.

18. Y. Zhang, F. M. Heim, N. Song, J. L. Bartlett and X. Li, ACS Energy Lett., 2017, 2, 2696.

19. Y. Mao, G. Li, Y. Guo, Z. Li, C. Liang, X. Peng and Z. Lin, Nat. commun., $2017,8,14628$.

20. A. M. Hafez, Y. Jiao, J. Shi, Y. Ma, D. Cao, Y. Liu and H. Zhu, Adv. Mater., 2018, 30, 1802156.

21. G. Huang, J. Han, F. Zhang, Z. Wang, H. Kashani, K. Watanabe and M. Chen, Adv. Mater., 2018, 1805334.

22. Y. Sun, G. Zheng, Z. W. Seh, N. Liu, S. Wang, J. Sun, H. R. Lee, Y. Cui, Chem 2016, 1, 287.

23. L. Liu, Y. X. Yin, J. Y. Li, N. W. Li, X. X. Zeng, H. Ye, Y. G. Guo and L. J. Wan, Joule, 2017, 1, 563.

24. W. Long, B. Fang, A. Ignaszak, Z. Wu, Y. J. Wang and D. Wilkinson, Chem. Soc. Rev., 2017, 46, 7176.

25. Z. Sun, S. Jin, H. Jin, Z. Du, Y. Zhu, A. Cao, H. Ji and L. J. Wan, $A d v$ Mater., 2018, 1800884.

26. S. Jin, S. Xin, L. Wang, Z. Du, L. Cao, J. Chen, X. Kong, M. Gong, J. Lu and Y. Zhu, Adv. Mater.,2016, 28, 9094.

27. I. Derr and C. Roth, "Countering Degradation of Carbon Felt Electrodes in All-Vanadium Redox Flow Batteries (VRFB)", presented at Meeting Abstracts, 2016.

28. T. C. Chang, J. P. Zhang and Y. K. Fuh, J. Power Sources, 2014, 245, 66.

29. Z. Liang, G. Zheng, C. Liu, N. Liu, W. Li, K. Yan, H. Yao, P. C. Hsu, S. Chu and Y. Cui, Nano lett., 2015, 15, 2910.

30. D. Lin, J. Zhao, J. Sun, H. Yao, Y. Liu, K. Yan and Y. Cui, P. Natl. A. Sci., 2017, 114, 4613-4618.

31. H. G. Schweiger, O. Obeidi, O. Komesker, A. Raschke, M. Schiemann, C. Zehner, M. Gehnen, M. Keller and P. Birke, Sensors, 2010, 10, 5604

32. W. Zhao, G. Luo and C. Y. Wang, J. Electrochem. Soc., 2015, 162, A1352.

Publisher's Note Engineered Science Publisher remains neutral with regard to jurisdictional claims in published maps and institutional affiliations. 